\title{
Global Selection of Saccadic Target Features by Neurons in Area V4
}

\author{
Brittany E. Burrows, ${ }^{1}$ Marc Zirnsak, ${ }^{1,2}$ Hessameddin Akhlaghpour, ${ }^{1}$ Megan Wang, ${ }^{1}$ and Tirin Moore ${ }^{1,2}$ \\ ${ }^{1}$ Department of Neurobiology, Stanford University School of Medicine and ${ }^{2}$ Howard Hughes Medical Institute, Stanford, California 94305
}

\begin{abstract}
Psychophysical and neurophysiological studies indicate that during the preparation of saccades, visual processing at the target location is facilitated automatically by the deployment of attention. It has been assumed that the neural mechanisms involved in presaccadic shifts of attention are purely spatial in nature. Saccade preparation modulates the visual responses of neurons within extrastriate area $\mathrm{V} 4$, where the responses to targets are enhanced and responses to nontargets are suppressed. We tested whether this effect also engages a nonspatial form of modulation. We measured the responses of area $\mathrm{V} 4$ neurons to oriented gratings in two monkeys (Macaca mulatta) making delayed saccades to targets distant from the neuronal receptive field (RF). We varied the orientation of both the RF stimulus and the saccadic target. We found that, in addition to the spatial modulation, saccade preparation involves a feature-dependent modulation of V4 neuronal responses. Specifically, we found that the suppression of area V4 responses to nontarget stimuli during the preparation of saccades depends on the features of the saccadic target. Presaccadic suppression was absent when the features of the saccadic target matched the features preferred by individual V4 neurons. This feature-dependent modulation occurred in the absence of any feature-attention task. We show that our observations are consistent with a computational framework in which feature-based effects automatically emerge from saccade-related feedback signals that are spatial in nature.
\end{abstract}

Key words: area V4; attention; cognition; oculomotor; visual perception; visual system

\section{Introduction}

Psychophysical and neurophysiological studies demonstrate a general bias in the visual processing of saccadic targets at the time of movements. For example, visual performance is facilitated at the endpoints of planned saccades, compared with other locations (Hoffman and Subramaniam, 1995; Deubel and Schneider, 1996). Correspondingly, saccades to stimuli within the receptive fields (RFs) of visual cortical neurons are preceded by an enhancement of stimulus-driven responses (Chelazzi et al., 1993; Moore et al., 1998; Khayat et al., 2004). In contrast, perceptual sensitivity at nontarget locations is reduced at the time of saccades (Ross et al., 2001), and parallel neurophysiological studies show that visual cortical neurons exhibit reduced sensitivity to orientation (Moore and Chang, 2009), luminance contrast (Han et al., 2009), and visual salience (Burrows and Moore, 2009) before saccades directed toward non-RF targets. Evidence of a bias toward the processing of saccadic targets over that of nontarget stimuli has generally been taken as evidence of an interdepen-

Received Feb. 26, 2013; revised April 2, 2014; accepted April 8, 2014.

Author contributions: B.E.B., M.Z., and T.M. designed research; B.E.B., M.Z., and H.A. performed research;B.E.B., M.Z., H.A., M.W., and T.M. analyzed data; B.E.B., M.Z., and T.M. wrote the paper.

This work was supported by National Institutes of Health EY014924, National Science Foundation IOB-0546891, the McKnight Foundation, and National Research Service Award F31MH081500 to B.E.B. We thank D. S. Aldrich for technical assistance and F. H. Hamker for valuable comments.

The authors declare no competing financial interests.

Correspondence should be addressed to Tirin Moore, Department of Neurobiology, Stanford University School of Medicine, Stanford, CA 94305. E-mail: tirin@stanford.edu.

DOI:10.1523/JNEUROSCI.0867-13.2014

Copyright $\odot 2014$ the authors $\quad 0270-6474 / 14 / 346700-07 \$ 15.00 / 0$ dence of spatial attention and saccadic preparation (Rizzolatti et al., 1987; Hoffman and Subramaniam, 1995; Deubel and Schneider, 1996; Moore et al., 2003). Moreover, it has been suggested that this bias reflects the causal role that gaze control mechanisms play in the deployment of spatially based attention (Rizzolatti et al., 1987; Moore et al., 2003; Knudsen, 2007), and there has been much experimental support of this possibility (Cavanaugh and Wurtz, 2004). At present, the role of gaze control mechanisms in spatially deployed visual attention is widely accepted.

In contrast to the apparent interdependence of saccadic preparation and spatially based attention, it is generally held that nonspatial attention, such as feature-based attention, involves different underlying mechanisms (Rizzolatti et al., 1987; Reynolds and Chelazzi, 2004). Furthermore, recent neurophysiological evidence seems to support this idea (Bichot et al., 2005; Hayden and Gallant, 2005). Unlike spatial attention, featurebased attention is spatially nonspecific, and instead involves the selective processing of specific stimulus features (Hayden and Gallant, 2005). Thus, an involvement of gaze control mechanisms, which are inherently spatial, would seem less likely. Nonetheless, some psychophysical and computational studies (Pollatsek et al., 1990; Jüttner and Röhler, 1993; Hamker, 2005) suggest the possibility of an influence of saccadic preparation on the processing of specific features in a spatially nonspecific manner. For example, Jüttner and Röhler (1993) found that subjects could more accurately report the orientation of a postsaccadic foveal stimulus when an identical stimulus is presented at the peripheral target 
Fixation

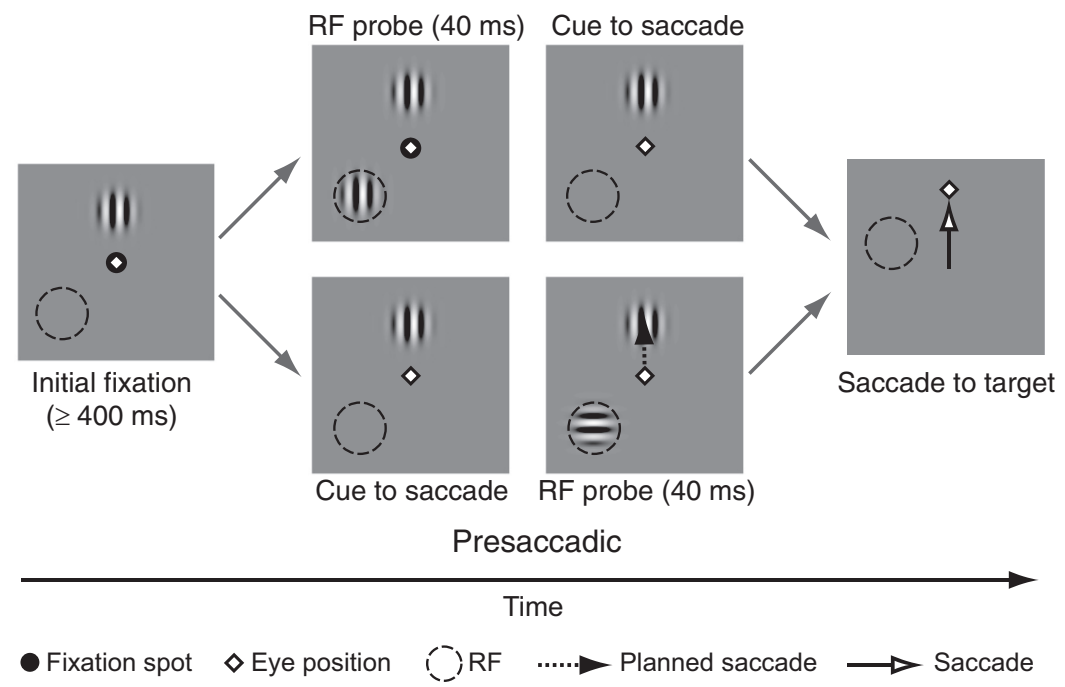

Fixation

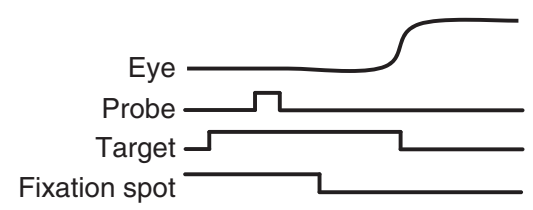

Presaccadic

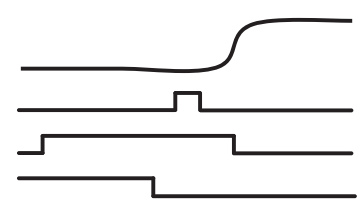

Figure 1. Measurement of target feature-dependent modulation in a delayed saccade task. In each trial, the monkey fixated (diamond) a central fixation spot (black circle) on the display, followed by the presentation of a saccade target. In the fixation condition (top), a probe stimulus appeared briefly in the RF while the monkey continued to fixate, followed by the cue to saccade (the removal of the fixation spot). In the presaccadic condition (bottom), the cue to saccade occurred before the probe, and the probe appeared just before saccade initiation. The saccadic target and the RF probe were both gratings, presented at either a neuron's preferred (P) or nonpreferred (NP) orientation. Event traces below depict the time lines of the trial events in the two conditions.

location of the impending saccade than when the presaccadic and postsaccadic stimuli are oriented differently. Moreover, this target feature-dependent bias in postsaccadic perception is observed even in instances in which the target is moved from its original spatial location during the saccade (Pollatsek et al., 1990), suggesting that the influence of a saccadic target on visual perception may be spatially global. Finally, the computational framework proposed by Hamker (2005) predicts that such a global target feature-dependent bias might arise automatically in the absence of any feature-specific task set. We tested this possibility in the current study.

\section{Materials and Methods}

Two male monkeys (Macaca mulatta) weighing 9 and $11 \mathrm{~kg}$ were used as subjects in these experiments. General surgical procedures have been described previously (Burrows and Moore, 2009). All surgical and experimental procedures were approved by the Stanford University Administrative Panel on Laboratory Animal Care and the consultant veterinarian, and were in accordance with National Institutes of Health Guide for the Care and Use of Laboratory Animals and Society for Neuroscience guidelines.

Electrophysiology. Recordings from single V4 neurons were made through a surgically implanted cylindrical titanium chamber $(20 \mathrm{~mm}$ diameter) overlaying the prelunate gyrus. Electrodes were lowered into the cortex through a stainless steel guide tube using a hydraulic microdrive (Narishige). Neuronal activity was recorded extracellularly with varnish-coated tungsten microelectrodes (FHC) of 0.2-1.0 $\mathrm{M} \Omega$ impedance (measured at $1 \mathrm{kHz}$ ). Extracellular waveforms were digitized and classified as single neurons using on-line templatematching (FHC). The classical RFs of V4 neurons were mapped in a separate behavioral paradigm in which oriented bars were swept across the display in eight different directions during fixation. While the activity of the recorded cell was monitored via an audio amplifier, the edges of the RF were plotted on a second monitor. All V4 RFs in this study were in the lower contralateral visual field with eccentricities between 2.5 and $5^{\circ}$. The orientation preference of each isolated V4 neuron was determined from sets of trials in which single oriented bars $\left(0^{\circ}, 45^{\circ}, 90^{\circ}\right.$, or $\left.135^{\circ}\right)$ were swept across the neuronal RF at one of the two directions orthogonal to bar orientation. The orientation yielding the greatest response was then chosen as the preferred $(\mathrm{P})$ orientation for the primary experiment; the orthogonal orientation was chosen as the nonpreferred (NP) orientation. We also computed a standard selectivity index between $\mathrm{P}$ and NP probes for the population of neurons (see below, Analyses). The mean selectivity index across all neurons was 0.16 , corresponding to a $28 \%$ average reduction in response to NP probes compared with $P$ probes.

Visual stimulation and behavioral procedures. Monkeys were seated in a primate chair in a quiet room and positioned in front of a visual display. Each monkey was trained to fixate a central spot on the display where stimuli were presented. Eye position was monitored via a scleral search coil, digitized, and stored at 500 Hz. Stimulus presentation, data acquisition, and behavioral monitoring were controlled by the CORTEX system. Stimuli were presented on a CRT display (Mitsubishi 2070SB-BK, 60 $\mathrm{Hz}$ ) at a resolution of $1024 \times 768$ pixels or on an LCD display (Samsung LN40B750, $60 \mathrm{~Hz}$ ) at a resolution of $1280 \times 1024$ pixels. The display was controlled by a Pentium-based computer with an NVIDIA FX5200 video card (CRT) or an ATI Radeon HD 3800 Series video card (LCD). Monkeys performed a task in which a Gabor grating (probe) was presented (40 ms) in the RF of a V4 neuron either during fixation or immediately before a saccade to another Gabor grating (target). The target appeared $7^{\circ}$ directly above the fixation spot and distant $\left(9-13^{\circ}\right)$ from the V4 RF. In the task, monkeys fixated a central spot for 100-200 $\mathrm{ms}$, after which the saccade target appeared and remained on for an extended delay while the monkey continued to fixate (Fig. 1). At the end of the delay period, the fixation spot was removed and the monkey was rewarded for making a saccade to the target. On half of the delayed saccade trials, the RF probe was presented before removal of the fixation spot (fixation condition), but following at least $400 \mathrm{~ms}$ of fixation in the presence of the saccade target. In the remaining saccade trials, the RF probe was presented following the removal of the fixation spot, but before the execution of the saccade (presaccadic condition). The behavioral conditions were randomly interleaved throughout each experimental session.

Analyses. Responses to visual stimuli were compared based on mean spike counts calculated within a $115 \mathrm{~ms}$ time window from the onset of the visual response in the population of neurons ( $55 \mathrm{~ms}$ after the RF probe) to the average offset of that response ( $170 \mathrm{~ms}$ after the RF probe). The duration of the time window was chosen to match the duration of the average response of all neurons to the brief $(40 \mathrm{~ms})$ probe stimulus. Neuronal responses to probe stimuli during the presaccadic conditions were taken only from trials on which the probe appeared between 120 and $40 \mathrm{~ms}$ before saccadic onset. The latter cutoff ensured that the probe stimulus was removed from the display before the onset of the saccade. 
The activity of each neuron on a given trial was normalized by dividing it by the maximum, single-trial response of that neuron across all conditions and trials. The normalized values were then averaged. Normalized averages were used to compute the selectivity index. The selectivity indices were computed as, $\left(\right.$ Response $_{\mathrm{P}}-$ Response $\left._{\mathrm{NP}}\right) /\left(\right.$ Response $_{\mathrm{P}}+$ Response $\left._{\mathrm{NP}}\right)$, where $\mathrm{P}$ and NP refer to the neuron's preference for the stimulus presented in the RF. Wilcoxon signed-rank tests were used for all statistical comparisons unless otherwise stated.

Model. To simulate the observed feature-dependent modulation of V4 neuronal responses during saccade preparation we used a previously described computational framework (Hamker, 2004, 2005; Hamker and Zirnsak, 2006; Zirnsak et al., 2011). While this framework (Hamker, 2004; Zirnsak et al., 2011) allows for an a priori feature-specific task set, and thus a global, feature-based top-down signal that biases V4 and inferotemporal (IT) neurons for specific stimulus attributes, it was not applied in the present simulations. A detailed mathematical description of the framework was described previously (Hamker, 2005; Hamker and Zirnsak, 2006). The simulation of V4 and IT follows Hamker and Zirnsak (2006) with the addition of a combined baseline and baseline-inhibition term (Hamker, 2005) to account for the spontaneous activity of the recorded V4 neurons.

\section{Results}

We studied the influence of saccadic target features on the responses of 128 neurons in macaque area V4 in two monkeys (48 in Monkey 1, 80 in Monkey 2). Using a delayed saccade task, we probed the responses of V4 neurons to a briefly flashed (40 ms) grating stimulus (probe) while the monkey was fixating or was preparing a saccade to a target presented distant from the neuron's RF (Fig. 1). The saccadic target was also a grating, and both it and the probe varied in orientation independently of one another. As a result, the two gratings were identical or orthogonal to one another, as well as identical or orthogonal to the preferred orientation of the neuron under study ( $\mathrm{P}$ or NP). By varying the state of saccadic preparation, as well as the probe and the target, we were thus able to examine the effects of each on V4 neuronal responses.

Our behavioral task required monkeys to make delayed saccades to targets for a reward. Thus, we first examined metrics of saccades to targets across the experimental conditions in both monkeys. Specifically, we examined the saccadic reaction time, i.e., the latency of saccade onset relative to the cue to move. Overall, saccadic reaction times were slightly, but significantly, greater during presaccadic probe conditions (Monkey 1: fixation = $260.6 \mathrm{~ms}$, presaccadic $=284.6 \mathrm{~ms}, p<10^{-12}$; Monkey 2: fixation $=209.4$, presaccadic $\left.=224.6, p<10^{-14}\right)$. The relative increase in reaction time for the presaccadic probe conditions is consistent with the remote distractor effect (Lévy-Schoen, 1969; Walker et al., 1995). However, the focus of our neurophysiological study was on the possible influence of target features, and their similarity to RF probes, on neuronal responses. Thus, we also compared saccades across variations in the target itself. We found no differences in reaction time between saccades made to targets that were presented at the preferred versus nonpreferred orientation of the recorded neurons $(p=0.10$ and $p=0.83$, fixation and presaccadic, respectively, Monkey $1 ; p=0.29$ and $p=0.18$, fixation and presaccadic, respectively, Monkey 2). We also found no differences between saccades made to targets when they matched $(M)$ versus did not match (NM) the RF probe stimulus ( $p=0.34$ and $p=0.35$, fixation and presaccadic, respectively, Monkey $1 ; p=0.40$ and $p=0.06$, fixation and presaccadic, respectively, Monkey 2). In addition, there were no differences in saccade duration across experimental conditions.
For the V4 neuronal data, we compared the responses of the full population of recorded neurons across the eight experimental conditions, which consisted of the fixation and presaccadic conditions and the four combinations of preferred and nonpreferred probe and target stimuli (Fig. $2 A$ ). In particular, we compared the responses to identical RF probes (either $\mathrm{P}$ or NP) between trials in which the target orientation was $\mathrm{P}$ or NP (Fig. 2B). For this comparison, the null hypothesis predicts equal presaccadic responses between these two conditions, and equal amounts of suppression, relative to the fixation condition. Note the overall negative differences in responses to presaccadic probe stimuli ( $\mathrm{P}$ and $\mathrm{NP}$ ) when saccades were directed to $\mathrm{P}$ targets versus NP targets. This difference reflected the dependence of the presaccadic response on the orientation of the target, which also influenced the degree to which presaccadic responses were suppressed relative to responses during fixation (Fig. 2C). That is, across the population of V4 neurons, we observed an overall significant suppression of responses in the presaccadic condition as compared with fixation ( $\mathrm{P}$ and NP probes: mean $\left._{\text {fixation-presaccadic }}=0.03, p<10^{-4}\right)$. However, the suppression was only significant when the saccadic target was presented at a nonpreferred orientation (NP targets). For saccades to NP targets, the response to the presaccadic probe was reliably reduced relative to that of the fixation condition for both $\mathrm{P}$ and NP probes (NP probe: mean $_{\text {fixation-presaccadic }}=0.07, p<10^{-3}$; $\mathrm{P}$ probe: mean $\left._{\text {fixation-presaccadic }}=0.06, p<10^{-4}\right)$. In contrast, there was no evidence of suppression when saccades were prepared to P targets (NP probe: mean $_{\text {fixation-presaccadic }}=0.012, p>$ 0.48 ; P probe: mean $\left._{\text {fixation }- \text { presaccadic }}=-0.02, p>0.91\right)$. Thus, the presaccadic suppression of probe responses depended significantly on the orientation of the saccadic target. In addition, regardless of the comparison to responses during the fixation condition, the presaccadic probe responses differed according to target type (P and NP probes: mean $_{(\text {Ptarget })-(\text { NPtarget })}=0.067, p<$ $0.0014), P$ targets yielding the greater probe responses. This difference was not present during the fixation condition ( $\mathrm{P}$ and NP probes: $\left.\operatorname{mean}_{(\text {Ptarget })-(\text { NPtarget })}=-0.0053, p>0.77\right)$.

Psychophysical evidence suggests that stimulus discrimination around the time of saccades may depend in part on the degree to which those stimuli match the features of saccadic targets (Jüttner and Röhler, 1993). Thus, we measured the degree to which features of the saccadic targets used in this study influenced the selectivity of V4 neurons. For both the fixation and presaccadic conditions, we computed a selectivity index for trials in which the saccadic target and the RF probe matched or did not match (Fig. 3A). (Selectivity indices were therefore computed from data points plotted diagonally from one another in Fig. 2C). During fixation, there was no difference between the $M$ and NM selectivity indices mean $\left._{\mathrm{M}-\mathrm{NM}}=-0.01, p>0.46\right)$. In contrast, during the presaccadic condition, the stimulus selectivity depended on whether the target and probe stimuli matched. Selectivity was greater by $\approx 39 \%$ when the probe and saccadic target matched compared with when they did not $\left(\operatorname{mean}_{\mathrm{M}-\mathrm{NM}}=0.06\right.$, $p<0.01)$. Thus the modest difference in suppression $(\approx 7 \%)$ observed presaccadically between $\mathrm{P}$ and NP targets resulted in a substantial difference in stimulus selectivity to the probes. In addition to the changes in presaccadic selectivity between the $M$ and NM conditions, we also observed a reduction in the degree to which the selectivity of one condition (e.g., NM) could predict that of the other (M) (Fig. 3B). During fixation, the M and NM selectivity were highly correlated across neurons $(r=0.85)$. In contrast, during the presaccadic condition, that correlation was significantly reduced ( $r=0.41$; Fisher $r$-to- $Z$ transformation, $p<$ 
A
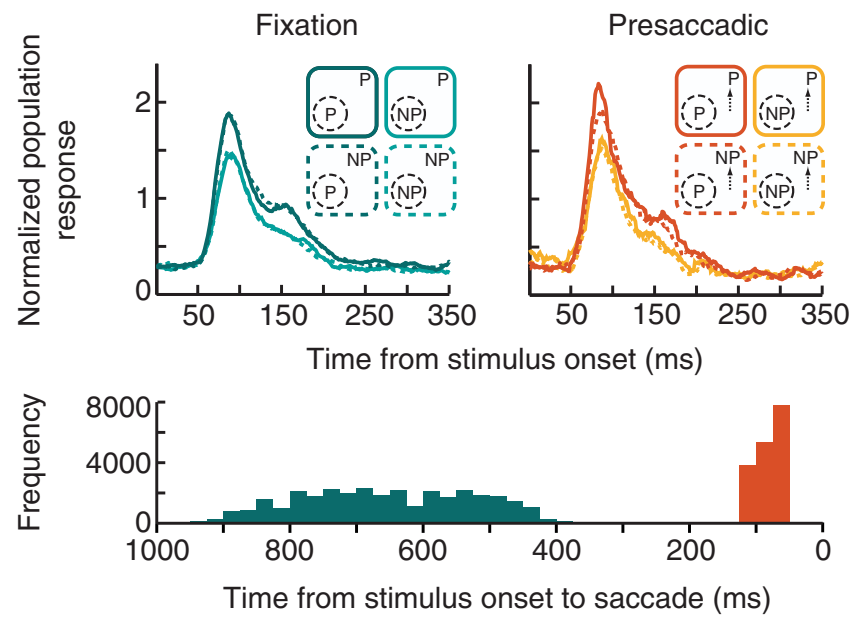

B

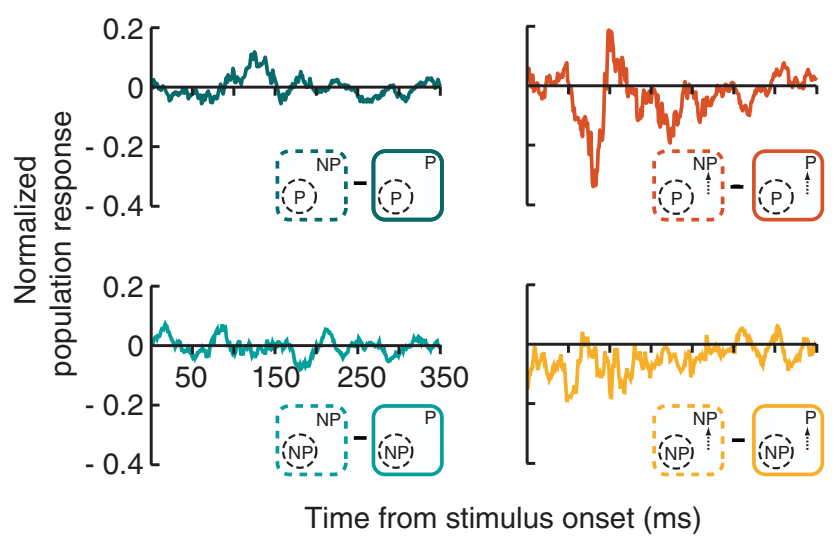

C
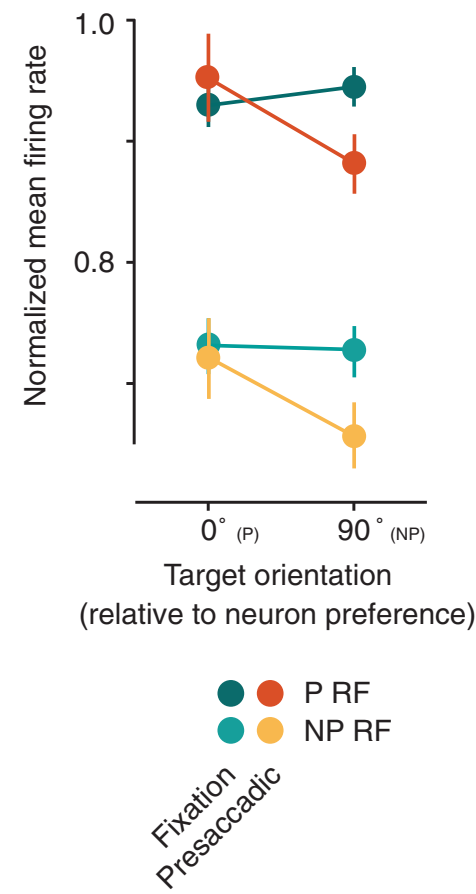

Figure 2. Target feature-dependent modulation of V4 neuronal responses. $\boldsymbol{A}$, Normalized population responses of all neurons ( $N=128$ ) to probe RF stimuli during fixation (left) and in the presaccadic period (right) are plotted for the four combinations of preferred (P) and nonpreferred (NP) probes and targets. Darker colors correspond to P RF stimuli. Bottom histogram shows the distributions of probe stimulus onset relative to saccade onset (0) for the fixation (left) and presaccadic period (right). $\boldsymbol{B}$, Difference in normalized population response between different target features (NP - P) for constant RF probe stimuli (top P, bottom NP). C, Normalized mean firing rates for all neurons during the fixation and presaccadic conditions plotted as a function of target feature (orientation) relative to each neuron's preference. Error bars denote SEM.

$0.001)$. Note that the decorrelation of presaccadic selectivities in the responses between $\mathrm{M}$ and NM stimuli is not a necessary outcome of the difference in selectivity. This result suggests that during the presaccadic period inputs driving responses are more distinct between the M and NM conditions than in the same conditions during fixation.

Next, we simulated our results using a computational framework modeling the dynamic interactions of visual and oculomotor areas (Hamker, 2005). This framework relies on well known connections (Felleman and Van Essen, 1991; Schall et al., 1995; Stanton et al., 1995) from downstream areas, such as the frontal eye field (FEF) and IT cortical areas (TEO and TE), to upstream visual areas including V4 and allows for feature-dependent (within IT) as well as space-dependent (within the FEF) gain modulations on model V4 neurons. The feature-specific feedback influences are depicted schematically in Figure $4 A$. During fixation, the tonic activity driven by the saccade target is insufficient to strongly bias IT neurons (Sheinberg and Logothetis, 2001). In this case, the feature-specific feedback signal to V4 neurons, including those processing the distant probe stimulus, is biased only slightly in favor of the target feature (Fig. 4B, Fixation). In the presaccadic period, however, the target bias in IT is stronger by virtue of a spatially selective mechanism acting on V4 and IT, presumably originating within oculomotor structures.
This spatial bias then results in a feature-specific feedback signal to V4 that is more strongly biased in favor of the target feature (Fig. 4B, Presaccadic). The observed presaccadic suppression of responses is due to an increased inhibitory drive, which is a function of the excitatory drive in the model, similar to normalization (Reynolds and Heeger, 2009). In the case of NP targets, neurons with preference $P$ receive more inhibition than excitation leading to a reduced presaccadic response as compared with fixation. For $\mathrm{P}$ targets, inhibition and excitation are relatively balanced for neurons preferring $\mathrm{P}$ leading to only small presaccadic response changes in the model.

\section{Discussion}

The responses of V4 neurons were suppressed when RF probes were presented presaccadically relative to when they were presented in the absence of a saccade plan. However, we found that the suppression only occurred when the saccadic target differed in orientation from that preferred by the recorded neuron (i.e., trials with NP targets). On trials in which the orientation of the saccadic target was preferred by the neuron (i.e., $\mathrm{P}$ targets), no suppression was observed. Thus, the suppression of responses to RF probes depended critically on the relationship between the orientation of the saccadic target and the orientation preference of V4 neurons. This target feature-dependent modulation re- 
A

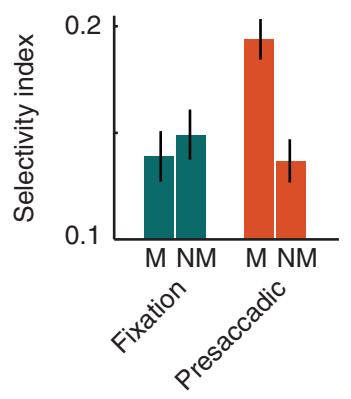

B

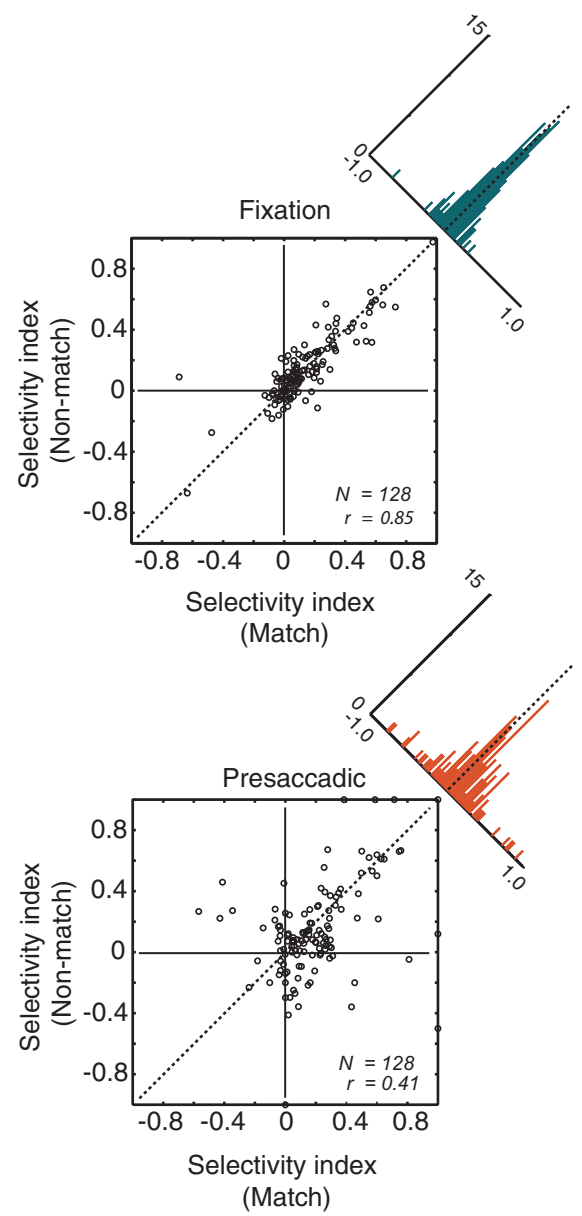

Figure 3. Target feature-dependent stimulus selectivity. $A$, Bar plot shows the mean selectivity indices for the fixation (left) and presaccadic (right) conditions, and for the match $(M)$ and non-match (NM) conditions for all neurons $(N=128)$. Selectivity indices were computed as the difference between responses to preferred (P) and nonpreferred (NP) orientations (right icon), normalized by their sum. $\boldsymbol{B}$, Scatter plots show the distributions and correlations between match and non-match selectivity during the fixation (top) and presaccadic (bottom) conditions. Diagonal histograms show the distribution of selectivity differences between the match and non-match conditions.

sulted in increased stimulus selectivity when the saccadic target and the probe matched versus when they did not.

The target feature-dependent effects we found are similar in nature to feature-based attention effects widely observed within extrastriate visual cortex, including area V4 (Treue and Martínez-Trujillo, 1999; Martinez-Trujillo and Treue, 2004; Bichot et al., 2005; Hayden and Gallant, 2005). Feature-based attention effects are observed in the context of tasks in which
A

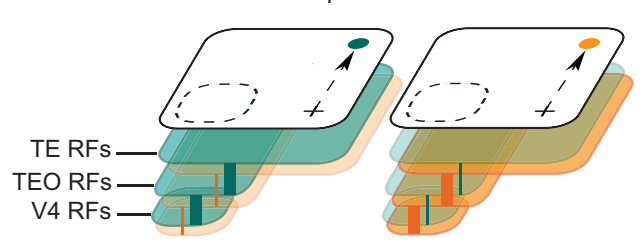

B
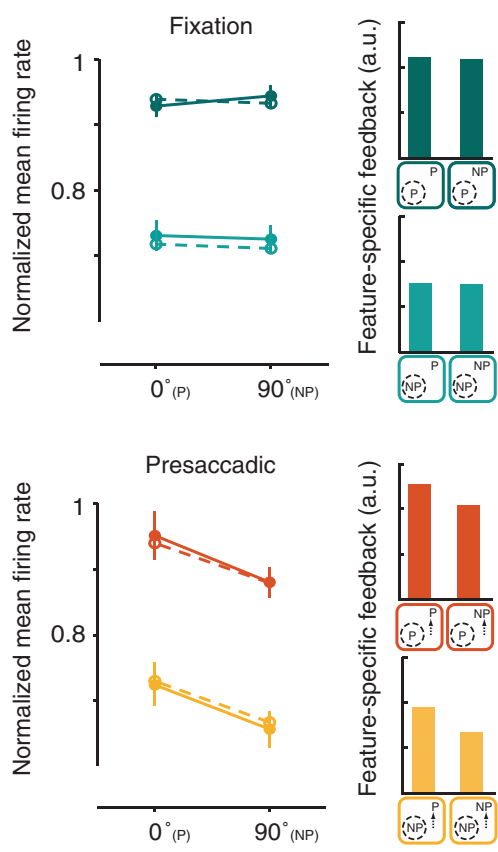

Target orientation (relative to neuron preference)

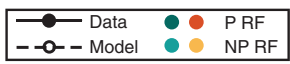

Figure 4. Modeling the emergence of feature-based effects during saccade preparation. $A$, Schematic depiction of a possible influence of reciprocal connections between V4 and downstream areas, such as in IT (areas TEO and TE) on presaccadic responses of V4 neurons. The effects of the selection of target features in areas where neurons have large RFs (i.e., TE) could spread via feedback to upstream areas with RFs distant from the target (i.e., V4). The two colors shown represent RFs of subpopulations of neurons preferring two different features, for example, blue and orange. The two presaccadic conditions shown depict instances in which the preparation of saccades to blue or orange targets biases the corresponding subpopulations; thicker lines and darker shading denote greater biases for that color. Presaccadic selection of saccade target features could originate from oculomotor structures providing spatial feedback, such as the FEF. B, Comparison of simulated (dashed) and recorded (solid) V4 responses during fixation and presaccadic periods of the delayed saccade task. Conventions are the same as in Figure 2. Simulated mean firing rates fell within the error bars of the experimental data in all cases. Bar plots depict the feature-specific feedback strength received by the model $\mathrm{V} 4$ neuron.

monkeys are trained to attend to (e.g., to search for) a particular stimulus feature independent of spatial location. For example, Bichot et al. (2005) found that when a cued search target is comprised of features preferred by the recorded neuron, responses to both preferred and nonpreferred RF stimuli are enhanced relative to trials in which the search target is not comprised of preferred features. This modulation occurs at all locations. The crucial difference between these studies and ours, however, is that we observed target feature-dependent effects without a feature attention task. Instead, we found that simply preparing a saccade to a visual target was sufficient to bring about a target featuredependent modulation of $\mathrm{V} 4$ responses. This result suggests that 
at least some component of the global selection of target features occurs by default during all visually guided saccades, and that it is not solely a phenomenon of feature-based attention. Given that most saccades are not planned within the context of visual search or other feature attention task, the global selection of target information during saccades we observed might play a more fundamental role in visual perception.

Previous studies suggest that feedback connections from saccade-related areas provide a means by which target features are selected and maintained across saccades (Moore et al., 1998; Hamker, 2004). During the preparation of saccades, spatial attention becomes anchored to the area of visual space occupied by the target (Hoffman and Subramaniam, 1995; Deubel and Schneider, 1996). Consistent with this psychophysical effect are the coincident presaccadic enhancements observed within saccade-related structures (Bruce and Goldberg, 1985) and visual cortex (Moore et al., 1998). A recent study provides the most direct evidence to date of an influence of neuronal activity within the former (e.g., FEF) on the latter (e.g., area V4; Noudoost and Moore, 2011). Feedback from saccade-related areas may not merely enhance the activity of V4 neurons at the location of the saccade target, but it might also bias $\mathrm{V} 4$ activity at more distant, nontarget locations in favor of target features. Such an outcome seems plausible if, for example, one considers that visually selective neurons in downstream areas have RFs that encompass both target and nontarget stimuli. For example, neurons in IT cortex have RFs that cover much of the visual field, are highly selective for complex visual stimuli (Desimone et al., 1984), and they are modulated during saccade preparation (Chelazzi et al., 1993; Sheinberg and Logothetis, 2001). One might expect that the presaccadic selection of target features by subpopulations of IT neurons would be conveyed to all neurons in upstream, more retinotopic visual areas (e.g., V4) via feedback connections, regardless of their RF position. As a result, an initial spatially based selection of target features during saccade preparation could give way to a global, feature-based bias in visual processing.

In addition, we showed that a class of computational models that assume feature-specific feedback connections between brain areas, as outlined above, predict the observed target featuredependent modulation (Hamker, 2005). The model also predicts that the observed effects in V4, in particular the presaccadic change in selectivity, should be stronger in more downstream areas such as TEO and TE. Moreover, while the influence of probe stimulus properties on suppression has been well documented in psychophysical studies (Burr et al., 1994; Michels and Lappe, 2004) our results and model point toward a more direct influence of the saccade target. In particular, manipulations of the saccade target that change the excitatory drive, and in turn the amount of inhibition, should lead to corresponding changes in both psychophysical and electrophysiological measurements of saccadic suppression. Our study suggests that spatial-based saccade preparation can facilitate the selection of target features throughout the visual field. As this selection appears to occur automatically, and without a feature-attentive task set, we speculate that it may facilitate the dominant perception of target features that appears to occur during saccadic eye movements (Pollatsek et al., 1990; Jüttner and Röhler, 1993; Deubel and Schneider, 1996). Moreover, our results suggest that this bias toward saccade target features might actually interfere with performance on feature attention tasks when saccades to nontarget features are involved.

\section{References}

Bichot NP, Rossi AF, Desimone R (2005) Parallel and serial neural mechanisms for visual search in macaque area V4. Science 308:529-534. CrossRef Medline

Bruce CJ, Goldberg ME (1985) Primate frontal eye fields. I. Single neurons discharging before saccades. J Neurophysiol 53:603-635. Medline

Burr DC, Morrone MC, Ross J (1994) Selective suppression of the magnocellular visual pathway during saccadic eye movements. Nature 371:511513. CrossRef Medline

Burrows BE, Moore T (2009) Influence and limitations of popout in the selection of salient visual stimuli by area V4 neurons. J Neurosci 29: 15169-15177. CrossRef Medline

Cavanaugh J, Wurtz RH (2004) Subcortical modulation of attention counters change blindness. J Neurosci 24:11236-11243. CrossRef Medline

Chelazzi L, Miller EK, Duncan J, Desimone R (1993) A neural basis for visual search in inferior temporal cortex. Nature 363:345-347. CrossRef Medline

Desimone R, Albright TD, Gross CG, Bruce C (1984) Stimulus-selective properties of inferior temporal neurons in the macaque. J Neurosci 4:2051-2062. Medline

Deubel H, Schneider WX (1996) Saccade target selection and object recognition: evidence for a common attentional mechanism. Vision Res 36: 1827-1837. CrossRef Medline

Felleman DJ, Van Essen DC (1991) Distributed hierarchical processing in the primate cerebral cortex. Cereb Cortex 1:1-47. CrossRef Medline

Hamker FH (2004) A dynamic model of how feature cues guide spatial attention. Vision Res 44:501-521. CrossRef Medline

Hamker FH (2005) The reentry hypothesis: the putative interaction of the frontal eye field, ventrolateral prefrontal cortex, and areas V4, IT for attention and eye movement. Cereb Cortex 15:431-447. CrossRef Medline

Hamker FH, Zirnsak M (2006) V4 receptive field dynamics as predicted by a systems-level model of visual attention using feedback from the frontal eye field. Neural Netw 19:1371-1382. CrossRef Medline

Han X, Xian SX, Moore T (2009) Dynamic sensitivity of area V4 neurons during saccade preparation. Proc Natl Acad Sci U S A 106:13046-13051. CrossRef Medline

Hayden BY, Gallant JL (2005) Time course of attention reveals different mechanisms for spatial and feature-based attention in area V4. Neuron 47:637-643. CrossRef Medline

Hoffman JE, Subramaniam B (1995) The role of visual attention in saccadic eye movements. Percept Psychophys 57:787-795. CrossRef Medline

Jüttner M, Röhler R (1993) Lateral information transfer across saccadic eye movements. Percept Psychophys 53:210-220. CrossRef Medline

Khayat PS, Spekreijse H, Roelfsema PR (2004) Correlates of transsaccadic integration in the primary visual cortex of the monkey. Proc Natl Acad Sci U S A 101:12712-12717. CrossRef Medline

Knudsen EI (2007) Fundamental components of visual attention. Annu Rev Neurosci 30:57-78. CrossRef Medline

Lévy-Schoen A (1969) Détermination et latence de la réponse oculomotrice 'a deux stimulus simultanés ou successifs selon leur excentricité relative. L’Anée Psychol 69:373-392.

Martinez-Trujillo JC, Treue S (2004) Feature-based attention increases the selectivity of population responses in primate visual cortex. Curr Biol 14:744-751. CrossRef Medline

Michels L, Lappe M (2004) Contrast dependency of saccadic compression and suppression. Vision Res 44:2327-2336. CrossRef Medline

Moore T, Chang MH (2009) Presaccadic discrimination of receptive field stimuli by area V4 neurons. Vision Res 49:1227-1232. CrossRef Medline

Moore T, Tolias AS, Schiller PH (1998) Visual representations during saccadic eye movements. Proc Natl Acad Sci U S A 95:8981-8984. CrossRef Medline

Moore T, Armstrong KM, Fallah M (2003) Visuomotor origins of covert spatial attention. Neuron 40:671-683. CrossRef Medline

Noudoost B, Moore T (2011) Control of visual cortical signals by prefrontal dopamine. Nature 474:372-375. CrossRef Medline

Pollatsek A, Rayner K, Henderson JM (1990) Role of spatial location in integration of pictorial information across saccades. J Exp Psychol Hum Percept Perform 16:199-210. CrossRef Medline 
Reynolds JH, Chelazzi L (2004) Attentional modulation of visual processing. Annu Rev Neurosci 27:611-647. CrossRef Medline

Reynolds JH, Heeger DJ (2009) The normalization model of attention. Neuron 61:168-185. CrossRef Medline

Rizzolatti G, Riggio L, Dascola I, Umiltá C (1987) Reorienting attention across the horizontal and vertical meridians: evidence in favor of a premotor theory of attention. Neuropsychologia 25:31-40. CrossRef Medline

Ross J, Morrone MC, Goldberg ME, Burr DC (2001) Changes in visual perception at the time of saccades. Trends Neurosci 24:113-121. CrossRef Medline

Schall JD, Morel A, King DJ, Bullier J (1995) Topography of visual cortex connections with frontal eye field in macaque: convergence and segregation of processing streams. J Neurosci 15:4464-4487. Medline

Sheinberg DL, Logothetis NK (2001) Noticing familiar objects in real world scenes: the role of temporal cortical neurons in natural vision. J Neurosci 21:1340-1350. Medline

Stanton GB, Bruce CJ, Goldberg ME (1995) Topography of projections to posterior cortical areas from the macaque frontal eye fields. J Comp Neurol 353:291-305. CrossRef Medline

Treue S, Martínez-Trujillo JC (1999) Feature-based attention influences motion processing gain in macaque visual cortex. Nature 399:575-579. CrossRef Medline

Walker R, Kentridge RW, Findlay JM (1995) Independent contributions of the orienting of attention, fixation offset and bilateral stimulation on human saccadic latency. Exp Brain Res 103:294-310. Medline

Zirnsak M, Beuth F, Hamker FH (2011) Split of spatial attention as predicted by a systems-level model of visual attention. Eur J Neurosci 33: 2035-2045. CrossRef Medline 